\title{
Case Report: Successful Treatment with Miltefosine of Severe New World Mucosal Leishmaniasis Caused by Leishmania guyanensis
}

\author{
Manuel Calvopina, ${ }^{1 \star}$ Sara Jijon, ${ }^{1}$ Esteban Serrano, ${ }^{2}$ and Hirotomo Kato ${ }^{3}$ \\ ${ }^{1}$ OneHealth Research Group, Carrera de Medicina, Facultad de Ciencias de la Salud, Universidad De Las Américas (UDLA), Quito, Ecuador; \\ ${ }^{2}$ Servicio de Otorrinolaringología, Hospital de Especialidades Eugenio Espejo, Quito, Ecuador; ${ }^{3}$ Division of Medical Zoology, Department of \\ Infection and Immunity, Jichi Medical University, Tochigi, Japan
}

\begin{abstract}
An 88-year-old man with mutilating mucosal leishmaniasis (ML) involving septal perforation, with granulomas in the pharynx and larynx, was treated with oral miltefosine, $50 \mathrm{mg}$ three times/day for 28 days. Miltefosine, an antineoplastic agent, is considered an alternative option for the treatment of ML, showing efficacies of $75-92 \%$ in Bolivia, Brazil, and Argentina. The patient denied having previous cutaneous $(C L)$ leishmaniasis, and no CL lesions were recognized by physical examination. Parasites obtained from mucosal lesions were identified by cytochrome $b$ gene sequencing as Leishmania guyanensis. Clinical cure was observed 2 months posttreatment, and no evidence of reactivation was observed in the 3-year follow-up. Adverse effects such as nausea, loss of appetite, and epigastric pain were experienced during treatment with miltefosine. There is a need for improved access to miltefosine in leishmaniasis-endemic areas of Latin America and a greater awareness of $\mathrm{ML}$ and its treatment among physicians working in endemic countries.
\end{abstract}

\section{INTRODUCTION}

American tegumentary leishmaniasis (ATL) is a group of zoonotic diseases caused by around 12 species of the genus Leishmania. There are two main clinical forms of ATL: cutaneous (CL) and mucocutaneous/mucosal leishmaniasis (ML). Mucosal leishmaniasis is a complication of CL where metastasis to the mucosal tissues of the mouth and upper respiratory tract occurs by lymphatic or hematogenous dissemination. It is the most serious and destructive form of ATL. Mucosal leishmaniasis is caused mainly by Leishmania braziliensis; however, it is also observed in patients infected by Leishmania guyanensis, Leishmania panamensis, Leishmania amazonensis, and Leishmania peruviana, with most cases diagnosed in Bolivia, Brazil, and Peru. ${ }^{1-3}$ In Ecuador, parasitological confirmed cases of $M L$ have been reported from the Amazon region ${ }^{4,5}$ and a few cases from the Pacific coast. ${ }^{6}$ In the Ecuadorian Amazon, the predominating species is $L$. braziliensis, whereas in the Pacific coast region it is L. guyanensis, as determined by DNA sequencing of cytochrome $b$ (cyt b). ${ }^{7}$ Mucosal lesions can present after several months to 20 or more years after a primary CL lesion. ${ }^{1}{ }^{H}$ However, about $15 \%$ of cases of ML give no previous history of CL. ${ }^{6,8,9}$ Nasal lesions are always present, with nodules and infiltration of the anterior cartilaginous septum; later, perforation of the septum occurs with collapse of the nose. The pharynx, palate, larynx, trachea, and upper lip can also be involved. In the final stage, there is severe mutilation, with destruction of the nose, pharynx, and larynx. ${ }^{1}$

Mucosal leishmaniasis almost never heals spontaneously, and in general, treatment failures and relapses are common. ${ }^{1,3}$ The recommended treatment for $\mathrm{ML}$ by the WHO, Pan American Health Organization, and Ecuadorian Ministry of Public Health is the pentavalent antimonials. ${ }^{10}$ In Ecuador, meglumine antimoniate is available as Glucantime ${ }^{\circledR}(20 \mathrm{mg}$ $\mathrm{SbV} / \mathrm{kg} /$ day for 28 days by parenteral route, Aventis SanofiPharma, Sao Paulo, Brazil). According to the PAHO expert

*Address correspondence to Manuel Calvopina, Universidad De Las Américas (UDLA), Av. Granados E11-41 y Colimies, Po Box 17-179788, Quito 170137, Ecuador. E-mails: manuelcalvopina@gmail.com or manuel.calvopina@udla.edu.ec committee (2013), miltefosine is an alternative option for the treatment of $\mathrm{ML}$ at a dosage of $1.5-2.5 \mathrm{mg} / \mathrm{kg} / \mathrm{day}$ for 28 days, with a maximum dose of $150 \mathrm{mg} /$ day. ${ }^{10}$ Miltefosine is an alkylphosphocholine analog originally developed as an antineoplastic agent. The U.S. Food and Drug Administration (reference ID: 3473277) has approved oral miltefosine (Impavido ${ }^{\circledR}-$ Knight Therapeutics, Montreal, Canada) for treatment of visceral, CL, and mucosal leishmaniasis caused by some Leishmania species (https://www.accessdata.fda.gov/ drugsatfda_docs/nda/2014/204684Orig1s000SumR.pdf). The drug efficacy varies according to the Leishmania species and the geographic location where the studies were performed..$^{11}$ Adverse effects include vomiting, nausea, kinetosis, headache, and transient elevation of creatinine and aminotransferase levels. ${ }^{12}$ At present, four studies have used miltefosine for $M L$, all in patients infected with $L$. Viannia subgenus, including $L$. guyanensis, $L$. braziliensis, and L. panamensis. ${ }^{13}$

Herein, we report a case of an 88-year-old man diagnosed with severe and destructive ML caused by the species L. guyanensis and successfully cured by oral miltefosine.

\section{CASE REPORT}

On admission to the otorhinolaryngology service of a tertiary care hospital in Quito, an otherwise healthy 88-year-old mestizo man presented with nasal septal ulceration and extensive granulomatous lesions in the pharynx with accompanying dysphonia. The patient was born in Chunchi, a high Andean village. At 20 years of age, he moved to Cumanda, a subtropical area located in the Pacific coastal region of the province of Chimborazo. For the past 15 years, he had been residing in Quito. He had a 10-year history of nasal mucosal lesions that began with pruritus, nasal discharge, bleeding, crusts, and obstruction. It progressed to septal perforation, with granulomas in the pharynx and larynx producing dysphonia. Collapse of the nose occurred because of cartilage mutilation. He was clinically diagnosed with bacterial and fungal infections, receiving treatment with several classes of antibiotics (quinolones such as ciprofloxacin and macrolides such as clarithromycin) and itraconazole (200 mg/day for 
3 months). Because the ulceration persisted and progressed, he was treated with fluconazole (150 mg/day for 6 months) but without any improvement. Hence, he sought medical help at the Hospital in Quito. During the interview, he denied having traveled to the Amazon or any other leishmaniasis-endemic country.

On hospitalization, an otorhinolaryngological examination of the nasal and oral cavities showed nasal mucosal erythema, bleeding crusts, and destruction of the septum and turbinates (Figure 1A). Numerous nodules and granulomas were observed in the pharynx (Figure 2A). The patient did not have lymphadenopathy, hepatosplenomegaly, or fever. No active skin lesions or scars were observed over any part of the body.

All blood tests for white blood cells, red blood cells, hemoglobin, and platelet counts were within the normal range. ELISA for HIV, VDRL, hepatitis B surface antigen and fungal culture were all negative. Fasting blood glucose, blood urea nitrogen (BUN), and serum creatinine were 105, 15.5, and 1.93 $\mathrm{mg} / \mathrm{dL}$, respectively. Serum levels of both alanine aminotransferase and aspartate aminotransferase (AST) were normal before, during, and after treatment with miltefosine. The leishmanin skin test was performed by intradermal injection in the forearm, and an area of induration measured $15 \mathrm{~mm} 48$ hours postinjection. Leishmanin solution was provided by PECET, Universidad de Antioquia, Medellin-Colombia (Cat.No. MSTA 90000, $5 \mathrm{~mL}$ ).

Histopathology of a biopsy taken from the border of the ulcerative lesion on the nasal mucosa showed an inflammatory infiltrate, characterized by leukocytes, neutrophils, and lymphocytes, with scarce macrophages infected with few Leishmania spp. amastigotes. No malignant cells were observed. Staining for fungus and mycobacteria was negative. The tissue material was spotted onto an FTA Classic Card (Whatman, Newton Center, MA), and part of the spotted sample was used as a template for polymerase chain reaction (PCR). Leishmanial cyt b was amplified by PCR, and the cyt b gene sequence was subjected to a phylogenetic analysis. ${ }^{7}$ The analysis identified $L$. guyanensis as the causative agent (Figure 3).

The patient received ambulatory oral miltefosine $2.5 \mathrm{mg} / \mathrm{kg} /$ day (Impavido $50 \mathrm{mg}$ capsules every 8 hours) for 28 days. Taking the medication with (fatty) food was recommended. Controls were carried out every week to record/evaluate any side effects and changes in the mucosal lesions. Nausea, loss of appetite, and epigastric pain were associated with the ingestion of miltefosine. Misoprostol (Cytotec ${ }^{\circledR} 800$ ug every 8 hours, Piramal Healthcare United, United Kingdom) was prescribed to control the epigastric pain. Two months after treatment, the patient returned for a control and no active lesions were observed (Figures 1B and 2B). Three years later, the patient was healthy and no leishmaniasis reactivation was observed. A written consent signed by the patient's sister was obtained to publish the case including the pictures of the lesions.

\section{DISCUSSION}

In the present case, oral miltefosine was shown to be effective in curing severe mucosal lesions caused by the New World $L$. guyanensis. In the four previous studies in which miltefosine was used to treat ATL ML, all showed good drug efficacy. Thus, studies in Bolivia showed a cure rate of $71 \%,{ }^{14}$ but increasing the period of therapy from 4 weeks to 6 weeks, the cure rate increased to $75 \% .{ }^{15}$ In Brazil, using oral miltefosine at $1.3-2 \mathrm{mg} / \mathrm{kg} /$ day for 28 days, $11 / 12$ patients with $\mathrm{ML}$ were cured at 90 days after treatment, and on examination after 4 years, 16/18 patients were considered cured. ${ }^{16}$ In a pilot study in Argentina, seven of eight patients with ML were cured using a dose of $2.5-3.3 \mathrm{mg} / \mathrm{kg} /$ daily. ${ }^{17}$

Not all Leishmania species are equally susceptible to miltefosine. There is a large natural variability of susceptibility to miltefosine among the various Leishmania species, as tested in vitro and in vivo. ${ }^{18-20}$ In the four prior clinical studies that were carried out, L. braziliensis was the predominant infecting species. ${ }^{14-17}$ In a systematic review published in 2013, at 6 months, a significant difference was noted in the rate of complete cure favoring miltefosine when compared with meglumine antimoniate for L. panamensis and L. guyanensis. ${ }^{13}$ In Ecuador, miltefosine has never been used to treat ML, and this is the first time to show here the efficacy of miltefosine in an infection due to L. guyanensis; in a case report of diffuse CL leishmaniasis caused by Leishmania mexicana, miltefosine failed to cure both clinically and parasitologically. ${ }^{21}$

The efficacy of miltefosine in $M L$ is also dependent on the severity of the mucosal lesions. In Bolivia, for a "mild" ML disease (i.e., affecting nasal skin and nasal mucosa), a drug efficacy of $83 \%$ was attained, whereas in severe disease
A

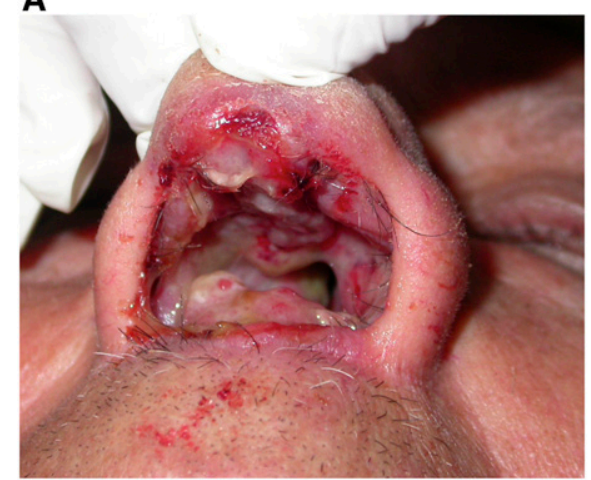

B

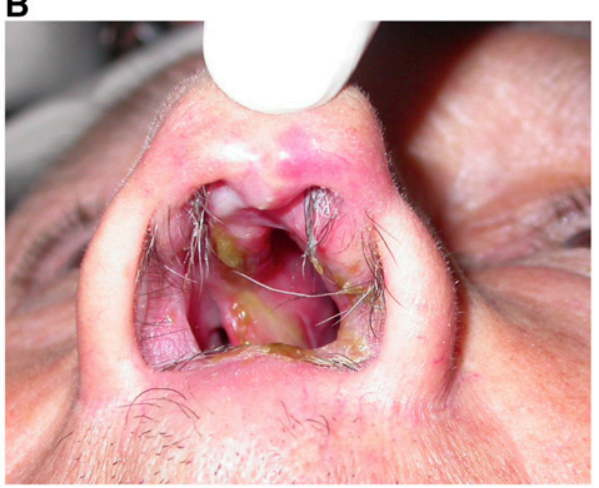

FIGURE 1. (A) Pretreatment. Nasal ulceration with septal perforation. Erythematous bleeding of ulcerative lesions of different sizes and bleeding crusts in the nostrils with complete septal mutilation. The presence of nodular lesions and hypertrophied turbinates. Ulcerations in the nostril mucosa and in the tip of the nose. The biopsy was taken from the mucosa of the tip of the nose. (B) Scarring of nostrils after 60 days of miltefosine treatment. No active lesions were observed in the nostrils and in the surrounding nasal mucosa and skin. Hypoplasic turbinates are seen. This figure appears in color at www.ajtmh.org. 
A

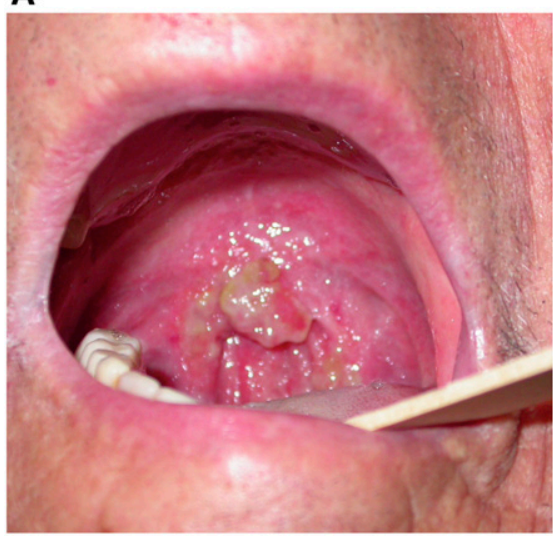

B

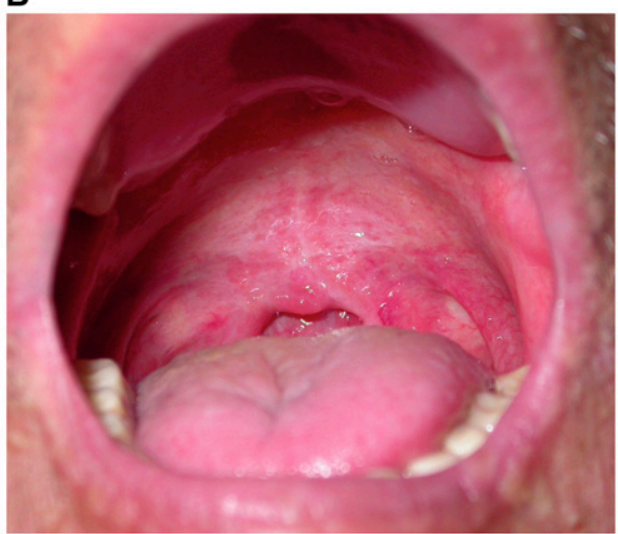

FIGURE 2. (A) Pretreatment of the oral cavity. Multiple granulomatous lesions of different sizes with active borders scattered throughout the patient's soft palate, posterior pillars, and pharynx. Uvula amputation. (B) Mucosa of the soft palate and posterior pillars are fully healed with only scar tissue remaining after 60 days of miltefosine treatment. No active granulomatous lesions were observed. This figure appears in color at www.ajtmh.org.

(involving the palate, pharynx, and larynx), it was only $58 \%$ effective. ${ }^{15}$ This corroborates a study we performed in Ecuador, but using itraconazole, a good response to treatment was associated with a short evolution of the disease and mild to moderate response in severe disease. ${ }^{5}$ Nevertheless, the present case was considered severe as shown in the figures before treatment with miltefosine.

Of interest, in this patient, L. guyanensis was identified as the causative species, which he probably contracted while living in the subtropical Pacific coast where L. guyanensis and L. panamensis are the dominant species. ${ }^{7}$ This region is considered endemic for CL, but where only a few as four cases of $\mathrm{ML}$ have been described. ${ }^{6}$ In Ecuador, L. panamensis and L. guyanensis are considered "benign" as they rarely metastasize to the mucosa. Moreover, our finding indicated that

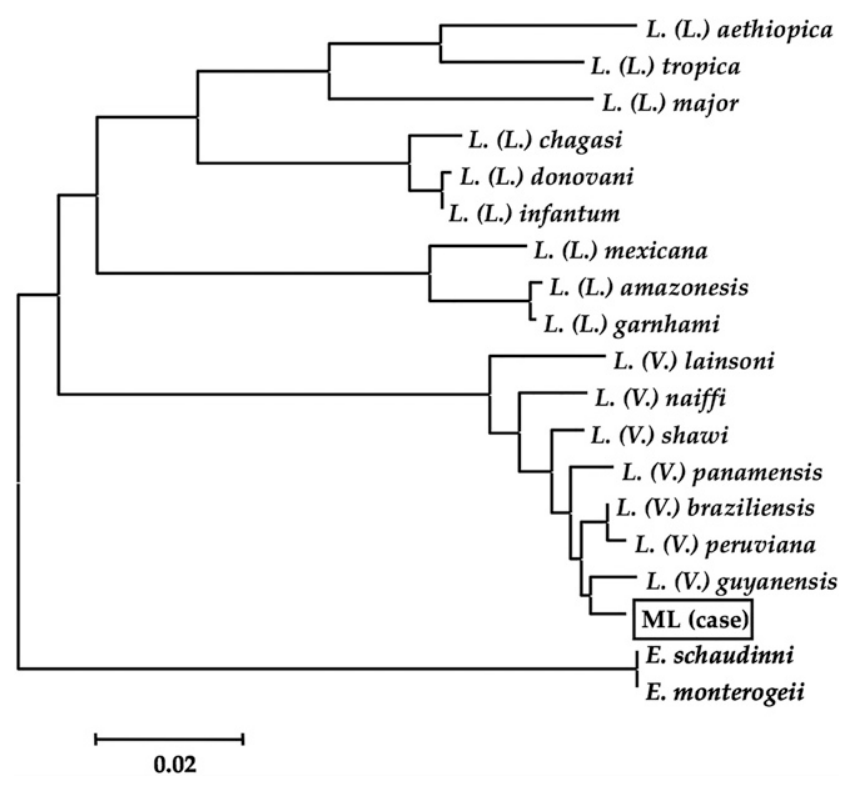

Figure 3. Phylogenetic tree of cytochrome b (cyt b) gene sequences from WHO reference Leishmania species. The leishmanial cyt $b$ genes were amplified from a mucosal lesion of the patient (sample MCL). Phylogenetic tree analysis with sequences obtained from the patient and those from 13 Leishmania spp. reference strains was performed. Scale bar indicates $0.01 \%$ divergence.
Ecuadorian L. guyanensis is capable of inducing $\mathrm{ML}$ as has been described in the neighboring countries of Colombia and Brazil. ${ }^{1}$ The localization of the mucosal lesions was similar to that reported elsewhere. ${ }^{8}$ Mucosal leishmaniasis cases detected in old patients are rare, but the present case was 88 years old; however, in a review of a retrospective study of 327 cases in Brazil, there was an increased risk above 60 years of age. In addition, there was a positive correlation between severity of mucosal disease and age. ${ }^{22}$

The fact that the patient did not have active skin lesions or scars is in accordance with previous studies of individuals having $M L, 2,6,8$ suggesting that a subclinical infection could occur. Furthermore, the ability of Leishmania parasites to remain latent during long periods and become active and invasive after (exogenous factors such as age) was demonstrated in the present case.,9 Five years after leaving the leishmaniasis-endemic area, while living in Quito, the mucosal symptomatology began to appear which has continued to progress in the last 10 years.

The standard 28-day miltefosine monotherapy regimen was well tolerated, except for mild gastrointestinal side effects, as described in several other clinical studies. ${ }^{18}$ It is important to note that after the 3-year follow-up period, there was still no clinical evidence of relapse of the mucosal lesions, although previous studies have reported a high relapse rate of $M L$ that tend to occur 1 year after completion of treatment. ${ }^{16}$ Miltefosine is not available in Ecuador, either in the public or in private sector, although in the CNMB 10th edition, it is considered as an alternative drug for leishmaniasis. There is a need for trials to compare safety and efficacy of miltefosine compared with antimonials in patients with ML in whom the infecting species of Leishmania have been identified.

Received November 23, 2019. Accepted for publication April 6, 2020. Published online June 8, 2020.

Acknowledgments: We thank Ivan Dario Velez from PECET, Universidad de Antioquia, Medellin-Colombia, for providing the Montenegro skin test antigen and miltefosine tablets. We thank Ronald Guderian for reviewing this case report.

Authors' addresses: Manuel Calvopina and Sara Jijon, OneHealth Research Group, Carrera de Medicina, Facultad de Ciencias de La Salud, Universidad De Las Américas (UDLA), Quito, Ecuador, E-mails: 
manuelcalvopina@gmail.com or manuel.calvopina@udla.edu.ec and sjijon@udlanet.ec. Esteban Serrano, Servicio de Otorrinolaringología, Hospital de Especialidades Eugenio Espejo, Quito, Ecuador, E-mail: serranoalmeida@yahoo.com. Hirotomo Kato, Division of Medical Zoology, Department of Infection and Immunity, Jichi Medical University, Tochigi, Japan, E-mail: hirok@jichi.ac.jp.

\section{REFERENCES}

1. WHO, 2010. Control of the Leishmaniases: Report of a Meeting of the WHO Expert Committee on the Control of Leishmaniases. Geneva, Switzerland: World Health Organization.

2. Osorio LE, Castillo CM, Ochoa MT, 1998. Mucosal leishmaniasis due to Leishmania (Viannia) panamensis in Colombia: clinical characteristics. Am J Trop Med Hyg 59: 49-52.

3. Guerra JA, Prestes SR, Silveira H, Coelho LI, Gama P, Moura A, Amato V, Barbosa M, Ferreira LC, 2011. Mucosal leishmaniasis caused by Leishmania (Viannia) braziliensis and Leishmania (Viannia) guyanensis in the Brazilian Amazon. PLoS Negl Trop Dis 5: e980.

4. Amunarriz M, 1991. Leishmaniasis. Estudios sobre Patologias Tropicales en la Amazonia Ecuatoriana. Napo, Ecuador: Editorial CICAME. Cap. 3, 41-64.

5. Calvopina M, Guevara AG, Armijos RX, Hashiguchi Y, Davidson RN, Cooper PJ, 2004. Itraconazole in the treatment of new world mucocutaneous leishmaniasis. Int $J$ Dermatol 43: 659-663.

6. Fernández TE, Almeida RT, 2012. Reporte de lesiones mucosas en leishmaniosis tegumentaria americana en el litoral (costa) ecuatoriano. Rev Patol Trop 41: 356-366.

7. Kato H, Gomez EA, Martini-Robles L, Muzzio J, Velez L, Calvopiña M, Romero-Alvarez D, Mimori T, Uezato H, Hashiguchi Y, 2016. Geographic distribution of Leishmania species in Ecuador based on the cytochrome B gene sequence analysis. PLOS Negl Trop Dis 10: e0004844.

8. Marsden PD, 1986. Mucosal leishmaniasis ("espundia" Escomel, 1911). Trans R Soc Trop Med Hyg 80: 859-876.

9. Machado-Coelho GL, Caiaffa WT, Genaro O, Magalhães PA, Mayrink W, 2005. Risk factors for mucosal manifestation of American cutaneous leishmaniasis. Trans $R$ Soc Trop Med Hyg 99: 55-61.

10. Pan American Health Organization, 2013. Leishmaniasis en las Américas: Recomendaciones Para el Tratamiento. Washington DC: OPS, 1-43.
11. Almeida OLS, Santos JB, 2011. Advances in the treatment of cutaneous leishmaniasis in the New World in the last ten years: a systematic review. An Bras Dermatol 86: 497-506.

12. Oliveira LF, Schubach AO, Martins MM, Passos SL, Oliveira RV, Marzochi MC, Andrade CA, 2011. Systematic review of the adverse effects of cutaneous leishmaniasis treatment in the New World. Acta Trop 118: 87-96.

13. Reveiz L, Maia-Elkhoury ANS, Nicholls RS, Sierra Romero GA, Yadon ZE, 2013. Interventions for American cutaneous and mucocutaneous leishmaniasis: a systematic review update. PLoS One 8: e61843.

14. Soto $\mathrm{J}$ et al., 2007. Treatment of Bolivian mucosal leishmaniasis with miltefosine. Clin Infect Dis 44: 350-356.

15. Soto J, Rea J, Valderrama M, Toledo J, Valda L, Ardiles J, Berman $\mathrm{J}, 2009$. Efficacy of extended (six weeks) treatment with miltefosine for mucosal leishmaniasis in Bolivia. Am J Trop Med Hyg 81: 387-389.

16. Sampaio RNR, Silva JSF, Paula CDR, Porto C, Motta JOC, Pereira LIA, Martins SS, Barroso DH, Freire GS, Gomes CM, 2019. A randomized, open-label clinical trial comparing the long-term effects of miltefosine and meglumine antimoniate for mucosal leishmaniasis. Rev Soc Bras Med Trop 52. e20180292.

17. Bustos MG, Barrio A, Parodi C, Beckar J, Moreno S, Basombrio $M, 2014$. Miltefosine versus meglumine antimoniate in the treatment of mucosal leishmaniasis [in Spanish]. Medicina ( $B$ Aires) 74: 371-377.

18. Dorlo TP, Balasegaram M, Beijnen JH, de Vries PJ, 2012. Miltefosine: a review of its pharmacology and therapeutic efficacy in the treatment of leishmaniasis. J Antimicrob Chemother 67: 2576-2597.

19. Yardley V, Croft SL, De Doncker S, Dujardin JC, Koirala S, Rijal S, Miranda C, Llanos-Cuentas A, Chappuis F, 2005. The sensitivity of clinical isolates of Leishmania from Peru and Nepal to miltefosine. Am J Trop Med Hyg 73: 272-275.

20. Croft SL, 2001. Monitoring drug resistance in leishmaniasis. Trop Med Int Health 6: 899-905.

21. Calvopina M, Gomez EA, Sindermann H, Cooper PJ, Hashiguchi $Y, 2006$. Relapse of new world diffuse cutaneous leishmaniasis caused by Leishmania (Leishmania) mexicana after miltefosine treatment. Am J Trop Med Hyg 75: 1074-1077.

22. Cincura C, de Lima CMF, Machado PRL, Oliveira-Filho J, Glesby MJ, Lessa MM, Carvalho EM, 2017. Mucosal leishmaniasis: a retrospective study of 327 cases from an endemic area of Leishmania (Viannia) braziliensis. Am J Trop Med Hyg 97: 761-766. 